\title{
New results on the parsec-scale properties of the Bologna Complete Sample
}

\author{
Gabriele Giovannini ${ }^{* a, b}$, Elisabetta Liuzzo ${ }^{a, b}$, Marcello Giroletti ${ }^{b}$, and Greg B. Taylor ${ }^{c}$ \\ ${ }^{a}$ Dipartimento di Astronomia di Bologna \\ via Ranzani 1, 40126 Bologna (I) \\ ${ }^{b}$ Istituto di Radioastronomia - INAF \\ via Gobetti 101, 40129 Bologna (I) \\ ${ }^{c}$ University of New Mexico \\ Albuquerque - NM (USA) \\ E-mail: ggiovann@ira.inaf.it, liuzzo@ira.inaf.it, \\ m.giroletti@ira.inaf.it, gbtaylor@unm.edu
}

We present an update of the parsec scale properties of the Bologna Complete Sample consisting of 95 radio sources with $\mathrm{z}<0.1$ from the B2 Catalog of Radio Sources and the Third Cambridge Revised Catalog (3CR). Thanks to new data obtained in phase reference mode, we have now parsec scale images for 76 sources of the sample. Most of them show a one-sided jet structure but we find a higher fraction of two-sided sources in comparison with previous flux-limited VLBI surveys. Results for two peculiar sources, 3C 293 and 3C 310, are presented and discussed in more detail.

The 9th European VLBI Network Symposium on The role of VLBI in the Golden Age for Radio Astronomy and EVN Users Meeting

September 23-26, 2008

Bologna, Italy

\footnotetext{
${ }^{*}$ Speaker.
} 


\section{Introduction}

The study of the parsec scale properties of radio galaxies is crucial to obtain information on the nature of their central engine. In order to study statistical properties of different classes of sources, it is necessary to define and observe a sample that is free from selection effects. To this aim, it is important to define the sample using a low radio frequency, to avoid observational biases related to orientation effects, thanks to the dominance at low radio frequency of the unbeamed extended emission. With this purpose, we started a project to observe a complete sample of radio galaxies (the Bologna Complete Sample (BCS)) selected within the B2 Catalog of Radio Sources and the Third Cambridge Revised Catalog (3CR) [6,7], with no selection constrain on the nuclear properties.

The sample consists of 95 radio sources. We selected the sources above a homogeneous flux density limit of $0.25 \mathrm{Jy}$ at $408 \mathrm{MHz}$ for the $\mathrm{B} 2$ sources and those above $10 \mathrm{Jy}$ at $178 \mathrm{MHz}$ for the 3CR sources [4] and applied the following criteria: 1) declination $>10^{\circ}$;2) Galactic latitude $|\mathrm{b}|$ $>15^{\circ}$; redshift $\mathrm{z}<0.1$.

At present VLBI observations are available for 76 sources. The missing 19 sources will require very sensitive observations (phase reference technique and large bandwidth) because of the very low activity of the radio core.

\section{Source morphology}

At parsec scale most of the sources (24 FR I and 7 FR II) show as expected a one-sided jet structure because of relativistic effects, however we have also a high number of sources with a symmetric jet structure. We classify as two sided all sources showing both a jet and counterjet, regardless of the value of the jet to the counterjet ratio or the length of the counterjet. The total number of sources with a two-sided morphology is 17 , corresponding to $\sim 25 \%$. This percentage is significantly higher than that found in previous samples in the literature: there are $11 \%$ symmetric sources in the PR (Pearson-Readhead) sample [10] and 4.6\% (19/411) in the combined PR and Caltech-Jodrell (CJ) samples [13, 12, 11].

For two-sided sources, the brightness ratio between the two jets is in the range 1-5, while most of the brightness lower limit in one-sided sources is higher than 5 confirming that the sensitivity in our maps is generally good enough to detect two sided sources. The main difference between the percentage of symmetric sources in the present sample and in previous samples is naturally explained in the framework of unified scheme models since our sources have been selected at low frequency and should not be affected by an orientation bias. The comparison between the VLA and VLBA jet position angles (P.A.) shows that most of the sources do not show a large misalignment and that the one-sided parsec scale (or the brigther jet in double-sided parsec jets) is oriented with respect to the nuclear emission, on the same side of the brighter kiloparsec-scale.

We compared the total flux at VLBA scale with the core arcsecond flux density. We find that $70 \%$ have a correlated flux density larger than $80 \%$ of the arcsecond core flux density. This means that in these sources we mapped most of the milliarcsecond (mas) scale structure and so we can properly connect the parsec to the kiloparsec structures. For 19 (30\%) sources, a significant fraction of the arcsecond core flux density is missing in the VLBA images. This suggest variability or the 


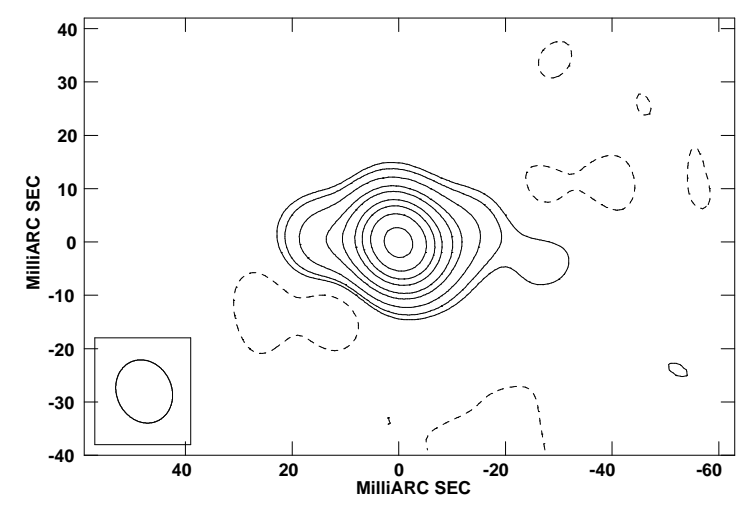

Figure 1: VLBA image at $1.7 \mathrm{GHz}$ of 3C 293. Contours are: -0.3 $0.30 .5123571015 \mathrm{mJy} / \mathrm{beam}$.

presence of significant structures between $\sim 30$ mas and 2 arcsec that the VLBA can miss because the lack of short baselines. To properly study these structures, future observations with EVLA or the e-MERLIN array will be necessary.

\section{Individual sources}

1350+31 - 3C293 This peculiar source has been studied in detail in the radio and optical bands. Recent results are discussed in [1] and [5], where the source structure is analyzed from the sub-arcsec to the arcmin scale. In Giovannini et al. 2005 ([8], we presented a VLBI image at 5 $\mathrm{GHz}$ where a possible symmetric two-sided jet structure is visible at mas resolution. Because of the complex inner structure we observed again this source with the VLBA at $1.7 \mathrm{GHz}$ in phase reference mode to properly map the inner region. Moreover we reduced VLA data in the A+ configuration (A configuration, adding also the VLBA-Pt telescope) to increase the VLA angular resolution and to study the connection between the sub-arcsec and the arcsec structure. In Fig. 1 we show the VLBI image of the core.

In agreement with the previous VLBA image at $5 \mathrm{GHz}$, the source shows a symmetric structure with two symmetric jets. The sub-arcsec VLA image at $5 \mathrm{GHz}$ shows the bright inner two-sided jet with a more external fainter diffuse emission (Fig. 2). The brightness distribution suggests a two phase activity: the source emission after some time re-started with a higher activity with respect to the older emission. However the jet direction appears to be constant in between the two activity epochs: after some oscillations the subarcsec jet is clearly oriented in E-W direction, and it moves to a PA $=-20$ (E side) and to a PA +20 (W side) in the direction of the arcsecond scale jet (Fig. 3).

Our suggestion is that this source is at a large angle with respect to the line of sight, and that oscillations and relatively small changes in the jet PA are present. In the region at about $2-3 \mathrm{kpc}$ from the core the Jet PA shows a large symmetric change with a kpc scale structure almost in NESE direction. This large change is not due to a change in the restarting nuclear activity but it looks constant in time and it is likely produced by the jet interaction with a rotating disk as discussed in [2]. 


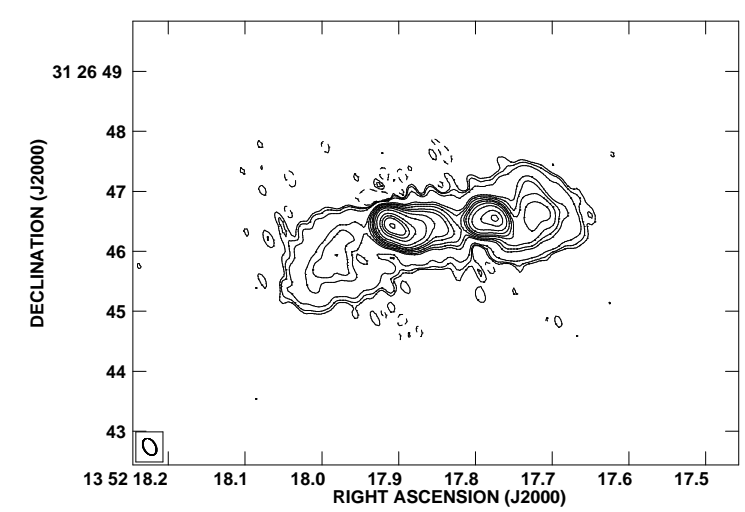

Figure 2: VLA+ (VLA in A configuration and VLBA-Pt) image at $5 \mathrm{GHz}$ of 3C 293. Levels are: -0.30 .3$ $0.51571015203050100150200300 \mathrm{mJy} / \mathrm{beam}$.

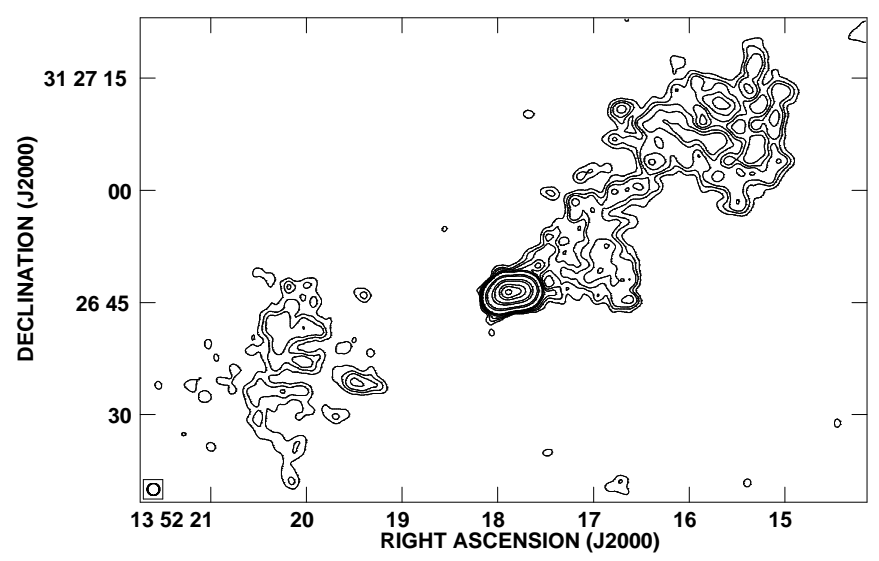

Figure 3: VLA image at $5 \mathrm{GHz}$ (B and C configuration) of 3C 293. Levels are: 0.10 .20 .30 .50 .7151050 $100300500800 \mathrm{mJy} / \mathrm{beam})$

1502+26-3C310 It is identified with a 15 mag elliptical galaxy at $z=0.0538$. In optical images this galaxy is flattened east-west, on an almost perpendicular direction to the radio jet axis. In the radio band at low resolution it appears as a relaxed double ([3]) with a steep radio spectrum $\left(\alpha_{750}^{26}=0.9 ; \alpha_{10.7}^{0.75}=1.4\right)$.

Low brightness lobes show a complex structure with filaments and bubbles. There is an unresolved radio core with a faint extension from the core to the North, extended 5", possibly identified as a faint jet ([3]). The VLBA structure is very complex. In [8] we showed an image interpreted as a one-sided emission in contrast with [9]. Because of the short observation and low flux density we observed again this source at 5 and $1.4 \mathrm{GHz}$ in phase reference mode. At $5 \mathrm{GHz}$ we have an elongated structure in E-W direction with a possible central nuclear structure and two symmetric jets or mini-lobes (Fig. 4). At lower resolution (Fig. 5) a faint emission is visible in the $\mathrm{N}$ direction, at the 


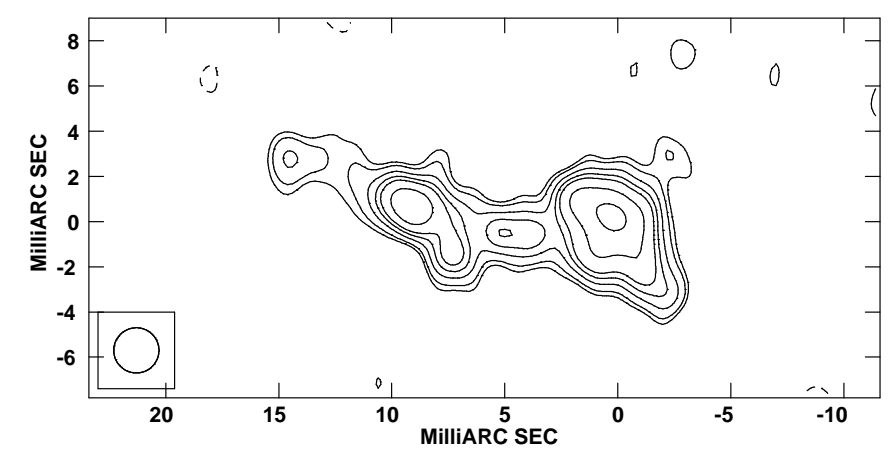

Figure 4: VLBA image at $5 \mathrm{GHz}$ of 3C 310. Levels are: $-0.20 .150 .20 .30 .40 .71 \mathrm{mJy} / \mathrm{beam}$

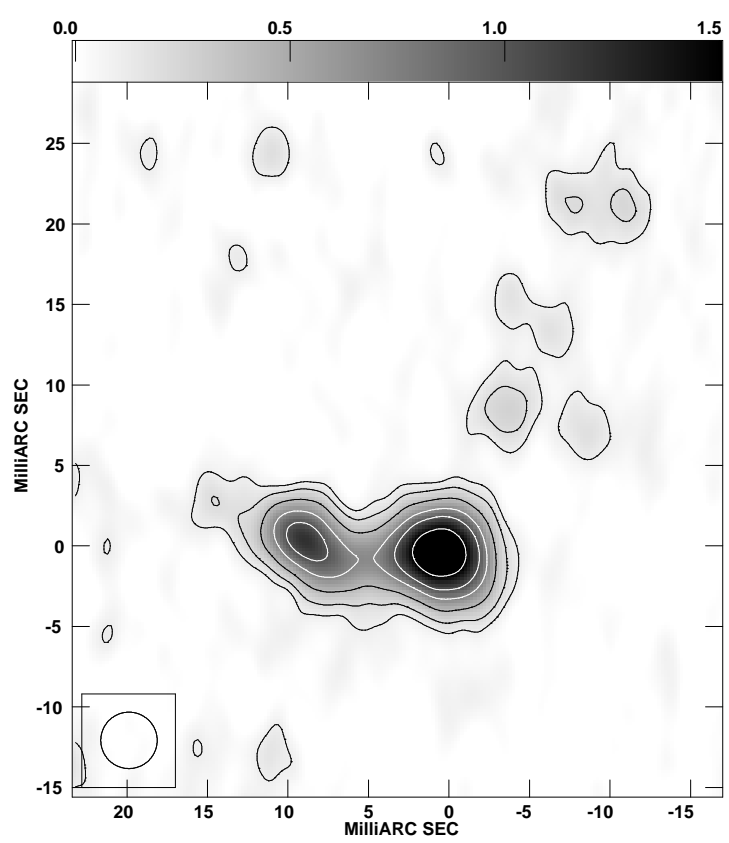

Figure 5: Low resolution VLBA image at $5 \mathrm{GHz}$ of 3C 310. Levels are: $0.10 .20 .40 .711 .5 \mathrm{mJy} / \mathrm{beam}$.

sampe PA of the kpc scale structure. At $1.4 \mathrm{GHz}$ the E-W structure is confirmed (Fig. 6), as well as the extension to the North in agreement with [9]. We produced images at 5 and $1.4 \mathrm{GHz}$ at the same resolution and cellsize and obtained a spectral index image. The spectral index distribution confirms the identification of the core with the faint emission at the center of the pc scale structure which shows a symmetric two-sided extension with a very steep spectral index.

The low resolution images suggest that after a few mas there is large change in the source PA from E-W to N-S in agreement with the kpc scale. This peculiar morpholoy could be related to 


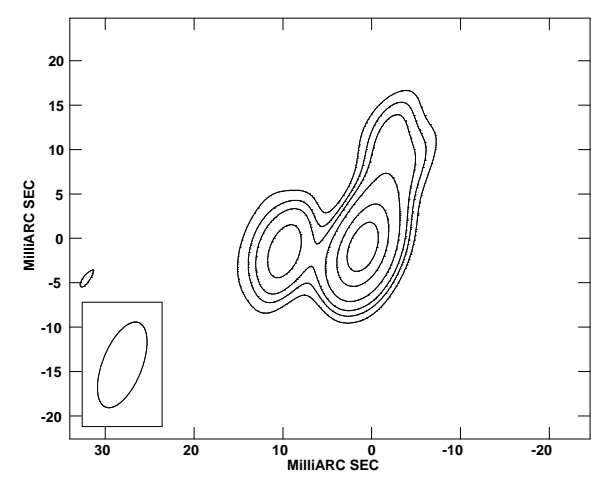

Figure 6: VLBA image at 1.6 GHz of 3C 310. Levels are: -1 $11.52357 \mathrm{mJy} / \mathrm{beam}$.

the interaction with a rotating disk as in $3 \mathrm{C} 293$ or to the presence of a double nucleus. In any case the pc scale structure visible in Fig. 4 and the large change in the radio structure PA suggest a sub-relativistic velocity for the two-sided emission.

\section{Acknowledgments}

The National Radio Astronomy Observatory is operated by Associated Universities, Inc., under cooperative agreement with the National Science Foundation.

\section{References}

[1] Beswick, R.J., Peck, A.B., Taylor, G.B., Giovannini,G. 2004 MNRAS 352, 49

[2] van Breugel, W., Heckman, T., Butcher, H., Miley, G. 1984 ApJ 277, 82

[3] van Breugel, W., and Fomalont, E. B. 1984 ApJ 282, 55

[4] Feretti, L., Giovannini, G., Gregorini, L., Parma, P., Zamorani, G. 1984, A\&A 139, 55

[5] Floyd, D.J.E., Perlman, E., Leahy, J.P., Beswick,R. J., Jackson, N.J., Sparks, W.B., Axon, D.J., O'Dea, C.P. 2006 ApJ, 639, 23

[6] Giovannini, G., Feretti, L., Comoretto, G. 1990 ApJ, 358, 159

[7] Giovannini, G., Cotton, W.D., Feretti, L., Lara, L., Venturi, T. 2001 ApJ, 552, 508

[8] Giovannini, G., Taylor, G. B., Feretti, L., Cotton, W. D.. Lara, L., Venturi, T. 2005 ApJ 618, 635

[9] Gizani, N. A. B., Garrett, M. A., Leahy, J. P. 2002 Journ. of Astroph. and Astr. 23, 89

[10] Pearson T. J., Readhead A. C. S., 1988, ApJ 328, 114

[11] Peck, A. B., and Taylor, G. B. 2000 ApJ 534, 90

[12] Polatidis A. G., Wilkinson P.N., Xu W., Readhead A.C.S., Pearson T. J., Taylor G. B., Vermeulen R. C., 1995, ApJS, 98, 1

[13] Taylor G. B., Vermeulen R. C., Pearson T. J., Readhead A. C. S., Henstock D. R., Browne I. W. A., Wilkinson P. N., 1994, ApJS 95, 345 